\title{
Antropometría de escolares del altiplano de Tarapacá (Comuna de Colchane, I Región): Contribución al estudio de la morfología humana
}

Luis GaVILÁN ${ }^{1}$ Y Estanislao AlvareZ ${ }^{2}$

\section{Introducción}

El conocimiento morfológico del hombre es de importancia fundamental en la cuantificación y cualificación de parámetros que permitan definir caracteres y rasgos, procesos y transformaciones propias del individuo. Asumimos con la literatura científica pertinente que el desarrollo morfológico $\mathrm{y}$ funcional del ser humano es producto de su condición biogenética y de los factores mesológicos a que está sometido.

El medio en que se desenvuelven los niños andinos aquí considerados posee características comunes a toda la región altiplánica. Este presenta condiciones difíciles para su desarrollo, las que han podido ser enfrentadas sólo a condición de una adecuada adaptación a ese hábitat. En la actualidad se plantea que existe una relación proporcional entre el medio ambiente altiplánico y la constitución anatomofisiológica del hombre andino que puede detectarse en términos morfológicos.

Los aymara del norte de Chile presentan características físicas y culturales particulares que los diferencian de las poblaciones urbanas y aun de otras rurales con diferentes modelos de desarrollo (en sentido ecológico). Esta premisa permite suponer que los rasgos somatológicos de los niños altiplánicos de la Comuna de Colchane deben también presentar rasgos físicos particulares o diferentes a otras poblaciones.

Con todo, es nuestro interés ayudar a establecer patrones morfológicos de la población escolar aymara que permitan deducir o adecuar fundamentos y técnicas de la práctica científica antropológica.

1 Programa Adaptación y Desarrollo Altiplánico. Sector Cariquima-Isluga, Juan Martínez 1906, Iquique, CHILE.

2 Pedro Vidal 1135, Depto. 31, Santiago, CHILE.

\section{Material y método}

Este estudio se realizó en la Comuna de Colchane, Provincia de Iquique, I Región de Tarapacá. La captación de la población se efectuó en los establecimientos educacionales correspondientes a la comuna. El universo está constituido por 188 niños, cuyas edades fluctúan entre seis y 10 años (informe proporcionado por la Oficina de Planificación y Estadística de la Secretaría Regional Ministerial de Educación, Iquique, agosto 1982). Sólo se omitieron aquellos individuos que no cumplieron con los requisitos necesarios para ingresar al estudio. Fueron examinados 137 alumnos, correspondiendo 72 hombres y 65 mujeres.

\begin{tabular}{|l|c|c|c|}
\hline \multicolumn{1}{|c|}{ Escuela } & Femenino & Masculino & Total \\
\hline Pisiga Choque & 6 & 14 & 20 \\
\hline Enquelga & 6 & 12 & 18 \\
\hline Escapiña & - & 6 & 6 \\
\hline Chijo & 8 & - & 8 \\
\hline Cariquima & 18 & 15 & 33 \\
\hline Mauque & 4 & 8 & 12 \\
\hline Colchane & 18 & 14 & 32 \\
\hline Villablanca & 5 & 3 & 8 \\
\hline Total & $\mathbf{6 5}$ & $\mathbf{7 2}$ & $\mathbf{1 3 7}$ \\
\hline
\end{tabular}

Cuadro 1. Distribución de la población escolar examinada entre 6 a 10 años, perteneciente a las escuelas de concentración y satélites de la Comuna de Colchane, Provincia de Iquique, 1982.

Las taxias antropométricas que se consideraron son: peso, talla total, talla sentado, perímetro cefálico, alto de cabeza, distancia biacromial, distancia bicrestal, longitud total de miembros superiores (derechoizquierdo) y longitud total de miembros inferiores (derecho-izquierdo).

Dada la importancia y característica, en este trabajo, de la variable nutricional, se confeccionó una ficha de encuesta alimentaria cuyo objetivo era agrupar 
la población según su aporte calórico diario. Como una manera de complementar la información que entregamos se seleccionaron una serie de marcadores genéticos, con lo cual se establecieron patrones que permitieron agrupar la población según se ascendencia étnica. Se ha pretendido controlar la variable socioeconómica a través del método de clasificación social de Graffar (1956), adaptado a la realidad chilena por el Centro de Crecimiento y Desarrollo de la Facultad de Medicina Norte de la Universidad de Chile.

\section{Análisis de datos}

Los datos antropométricos y antecedentes genéticos y ambientales se clasificaron y procesaron con diferentes métodos estadísticos, medidas de resúmenes, inferencias y correlación, entre otros. Para la confección de tablas, a cada individuo se le calculó la edad en días a partir de la fecha de nacimiento hasta el día del examen, incorporándose luego al intervalo de edad correspondiente.

A cada factor en estudio se asignaron diferentes categorías de acuerdo a premisas previamente establecidas. El factor genético se identificó con tres categorías: individuos con antecedentes de ascendencia caucasoide, individuos con antecedentes de ascendencia mongoloide e individuos con antecedentes de ascendencia mixta. Al factor nutricional o ingesta calórica se asignaron dos categorías: 1) niños internos y 2) niños externos. El factor socioeconómico se identificó con las categorías o estratos determinados por el test de Graffar.

\begin{tabular}{|l|c|c|c|c|}
\hline \multirow{2}{*}{ Edad (días) } & \multicolumn{2}{|c|}{ Grupo 1 (internos) } & \multicolumn{2}{c|}{ Grupo 2 (externos) } \\
\cline { 2 - 5 } & $\mathbf{N}$ & $\mathbf{\%}$ & $\mathbf{N}$ & $\mathbf{\%}$ \\
\hline $2190-2373$ & - & - & 10 & 100 \\
\hline $2374-2557$ & - & - & 16 & 100 \\
\hline $2558-2741$ & - & - & 19 & 100 \\
\hline $2742-2925$ & - & - & 18 & 100 \\
\hline $2926-3109$ & - & - & 7 & 100 \\
\hline $3110-3293$ & - & - & 18 & 100 \\
\hline $3294-3477$ & - & - & 10 & 100 \\
\hline $3478-3661$ & - & - & 17 & 100 \\
\hline $3662-3845$ & 1 & 8.3 & 11 & 91.6 \\
\hline $3846-4008$ & 5 & 50 & 5 & 50 \\
\hline Total & $\mathbf{6}$ & $\mathbf{4 . 4}$ & $\mathbf{1 3 1}$ & $\mathbf{9 5 . 6}$ \\
\hline
\end{tabular}

Tabla 1. Distribución de los escolares según edad y niveles de ingesta calórica diaria.

\section{Comentario}

El mayor porcentaje de la población estudiada se encuentra en el grado 2 de ingesta calórica diaria correspondiente a los niños escolares externos. Constituye $95.6 \%$ de la población examinada. Estos individuos reciben desayuno y almuerzo en las escuelas; la comida (cena) la realizan en sus respectivas casas.

Estimación : $1601 \mathrm{kcal} / \mathrm{día}$

Distribución : desayuno $=300 \mathrm{kcal} /$ día

almuerzo $=500 \mathrm{kcal} / \mathrm{día}$

cena $=801 \mathrm{kcal} / \mathrm{día}$

El grado 1 de ingesta calórica corresponde a los escolares internos. Estos individuos, que constituyen 4.4\% de la población examinada, reciben su alimentación completa en las escuelas de concentración de la Comuna.

Estimación : $2082 \mathrm{kcal} /$ día ;

Distribución : desayuno $=416 \mathrm{kcal} / \mathrm{día}$

almuerzo $=833 \mathrm{kcal} / \mathrm{día}$

cena $=833 \mathrm{kcal} / \mathrm{día}$

\begin{tabular}{|c|c|c|c|c|c|c|c|c|}
\hline \multirow{2}{*}{ Edad (días) } & \multicolumn{2}{|c|}{ Graffar 1-2 } & \multicolumn{2}{|c|}{ Graffar 3} & \multicolumn{2}{|c|}{ Graffar 4} & \multicolumn{2}{|c|}{ Graffar 5} \\
\hline & $\mathbf{N}$ & $\%$ & $\mathbf{N}$ & $\%$ & $\mathbf{N}$ & $\%$ & $\mathbf{N}$ & $\%$ \\
\hline $2190-2373$ & - & - & - & - & - & - & 10 & 100 \\
\hline $2374-2557$ & - & - & - & - & - & - & 16 & 100 \\
\hline $2558-2741$ & 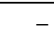 & - & - & - & - & - & 19 & 100 \\
\hline $2742-2925$ & - & - & - & - & - & - & 18 & 100 \\
\hline $2926-3109$ & - & - & - & - & - & - & 7 & 100 \\
\hline $3110-3293$ & - & - & - & - & - & - & 18 & 100 \\
\hline $3294-3477$ & - & - & - & - & - & - & 10 & 100 \\
\hline $3478-3661$ & - & - & - & - & - & - & 17 & 100 \\
\hline $3662-3845$ & - & - & - & - & - & - & 12 & 100 \\
\hline $3846-4008$ & - & - & - & - & - & - & 10 & 100 \\
\hline Total & - & - & - & - & - & - & 137 & 100 \\
\hline
\end{tabular}

Tabla 2. Distribución de los escolares según edad y nivel socioeconómico.

\section{Comentario}

En esta tabla observamos que $100 \%$ de los niños encuestados se ubican en el grado 5 del test de Graffar que corresponde al nivel socioeconómico bajo. Destacamos el hecho de que si bien estos datos dan a este trabajo validez comparativa, nos asociamos al pensamiento siguiente:

“...sin embargo, dada la estructura comunitaria y la igualdad de actividades productivas casi no encontramos en Isluga estratificación social. La 
mayor posesión de bienes (ganado y tierra o que podría suponerse establecería diferencias de estatus) no tiene en la práctica un efecto estratificador" (Provoste 1977: 5).

\begin{tabular}{|l|c|c|c|c|c|c|}
\hline \multirow{2}{*}{ Edad } & \multicolumn{2}{|c|}{$\begin{array}{c}\text { Ascendencia } \\
\text { caucasoide }\end{array}$} & \multicolumn{2}{c|}{$\begin{array}{c}\text { Ascendencia } \\
\text { mixta }\end{array}$} & \multicolumn{2}{c|}{$\begin{array}{c}\text { Ascendencia } \\
\text { mongoloide }\end{array}$} \\
\cline { 2 - 7 } & $\mathbf{N}$ & $\mathbf{\%}$ & $\mathbf{N}$ & $\mathbf{\%}$ & $\mathbf{N}$ & $\mathbf{\%}$ \\
\hline $2190-2373$ & - & - & 10 & 100 & - & - \\
\hline $2374-2557$ & - & - & 16 & 100 & - & - \\
\hline $2558-2741$ & - & - & 19 & 100 & - & - \\
\hline $2742-2925$ & - & - & 18 & 100 & - & - \\
\hline $2926-3109$ & - & - & 7 & 100 & - & - \\
\hline $3110-3293$ & - & - & 18 & 100 & - & - \\
\hline $3294-3477$ & - & - & 10 & 100 & - & - \\
\hline $3478-3661$ & - & - & 16 & 94.1 & 1 & 5.8 \\
\hline $3662-3845$ & - & - & 12 & 100 & - & - \\
\hline $3846-4008$ & - & - & 10 & 100 & - & - \\
\hline Total & - & - & $\mathbf{1 3 6}$ & $\mathbf{9 9 . 2}$ & $\mathbf{1}$ & $\mathbf{0 . 7}$ \\
\hline
\end{tabular}

Tabla 3. Distribución de los escolares según edad y origen étnico.

\section{Comentario}

Del total de los niños examinados $99.2 \%$ son de ascendencia genética mixta; $0.7 \%$ son de ascendencia genética mongoloide y no encontramos individuos de ascendencia genética caucasoide. Esta información debe entenderse en el marco del rango de probabilidad determinada por los marcadores genéticos seleccionados y utilizados. La frecuencia relativa de éstos se manifestó de la siguiente manera: $89 \%$ de la población estudiada presentó diente de pala; $16.1 \%$ úvula bífida; $58.3 \%$ dermatoglifos en torbellino; $0.8 \%$ tubérculo carabelli; $70.8 \%$ presentó trirradio C y $35.7 \%$ dermatoglifos de presilla.

\begin{tabular}{|l|r|r|c|c|c|c|}
\hline \multirow{2}{*}{ Edad (días) } & \multicolumn{2}{|c|}{ N } & \multicolumn{2}{c|}{ X (gr) } & \multicolumn{2}{c|}{ Ds } \\
\cline { 2 - 7 } & $\mathbf{M}$ & $\mathbf{F}$ & $\mathbf{M}$ & $\mathbf{F}$ & $\mathbf{M}$ & $\mathbf{F}$ \\
\hline $2190-2373$ & 4 & 6 & 29875 & 20500 & 2654 & 1958 \\
\hline $2374-2557$ & 9 & 7 & 21422 & 19957 & 1034 & 1923 \\
\hline $2558-2741$ & 6 & 13 & 25466 & 20846 & 3764 & 2117 \\
\hline $2742-2925$ & 11 & 7 & 23745 & 24143 & 2355 & 2474 \\
\hline $2926-3109$ & 2 & 5 & 23000 & 22300 & 1516 & 1029 \\
\hline $3110-3293$ & 10 & 8 & 25300 & 24312 & 1871 & 1983 \\
\hline $3294-3477$ & 5 & 5 & 26380 & 24500 & 3755 & 1852 \\
\hline $3478-3661$ & 10 & 7 & 27720 & 26757 & 1734 & 1664 \\
\hline $3662-3845$ & 7 & 5 & 28071 & 29960 & 3016 & 3163 \\
\hline $3846-4008$ & 9 & 2 & 30927 & 33500 & 2799 & 3500 \\
\hline
\end{tabular}

Tabla 4. Peso de escolares 6-10 años en relación a edad. Comuna de Colchane, 1982.

\section{Comentario}

En esta Tabla 4 se observa un incremento proporcionado del peso en relación a la edad. Si se comparan los resultados por sexo se evidencia que existen diferencias significativas entre uno y otro. En los primeros intervalos (seis y nueve años) correspondientes al sexo masculino el peso es superior al de las mujeres; sin embargo, esta característica se invierte en los intervalos finales, vale decir a los 10 años de edad.

\begin{tabular}{|l|c|c|c|c|c|c|}
\hline \multirow{2}{*}{ Edad (días) } & \multicolumn{2}{|c|}{ N } & \multicolumn{2}{c|}{ X $(\mathbf{m m})$} & \multicolumn{2}{c|}{ Ds } \\
\cline { 2 - 7 } & $\mathbf{M}$ & $\mathbf{F}$ & $\mathbf{M}$ & $\mathbf{F}$ & $\mathbf{M}$ & $\mathbf{F}$ \\
\hline $2190-2373$ & 4 & 6 & 1118 & 1097 & 44 & 40 \\
\hline $2374-2557$ & 9 & 7 & 1098 & 1075 & 35 & 43 \\
\hline $2558-2741$ & 6 & 13 & 1135 & 1116 & 16 & 45 \\
\hline $2742-2925$ & 11 & 7 & 1176 & 1160 & 51 & 46 \\
\hline $2926-3109$ & 2 & 5 & 1150 & 1138 & 20 & 20 \\
\hline $3110-3293$ & 10 & 8 & 1209 & 1191 & 52 & 39 \\
\hline $3294-3477$ & 5 & 5 & 1243 & 1219 & 61 & 64 \\
\hline $3478-3661$ & 10 & 7 & 1249 & 1257 & 42 & 48 \\
\hline $3662-3845$ & 7 & 5 & 1255 & 1288 & 44 & 60 \\
\hline $3846-4008$ & 8 & 2 & 1288 & 1446 & 68 & 14 \\
\hline
\end{tabular}

Tabla 5. Talla de escolares 6-10 años en relación a edad. Comuna de Colchane, 1982.

\section{Comentario}

La talla aumenta en relación a la edad. En los escolares de la Comuna de Colchane esta apreciación general se observa tanto en hombres como en mujeres. Se evidencia además que esta variable en los hombres entre seis y nueve años es superior a la de las mujeres. Esta característica se invierte en los intervalos etarios superiores (10 años).

\begin{tabular}{|l|r|r|c|c|c|c|}
\hline \multirow{2}{*}{ Edad (días) } & \multicolumn{2}{|c|}{$\mathbf{N}$} & \multicolumn{2}{c|}{ X (mm) } & \multicolumn{2}{c|}{ Ds } \\
\cline { 2 - 7 } & $\mathbf{M}$ & $\mathbf{F}$ & $\mathbf{M}$ & $\mathbf{F}$ & $\mathbf{M}$ & $\mathbf{F}$ \\
\hline $2190-2373$ & 4 & 6 & 508 & 498 & 14 & 13 \\
\hline $2374-2557$ & 9 & 7 & 506 & 509 & 15 & 8 \\
\hline $2558-2741$ & 6 & 13 & 509 & 505 & 7 & 9 \\
\hline $2742-2925$ & 11 & 7 & 514 & 511 & 13 & 13 \\
\hline $2926-3109$ & 2 & 5 & 512 & 513 & 12 & 14 \\
\hline $3110-3293$ & 10 & 8 & 525 & 516 & 16 & 11 \\
\hline $3294-3477$ & 5 & 5 & 520 & 525 & 11 & 20 \\
\hline $3478-3661$ & 10 & 7 & 520 & 520 & 10 & 7 \\
\hline $3662-3845$ & 7 & 5 & 528 & 520 & 15 & 10 \\
\hline $3846-4008$ & 8 & 2 & 531 & 536 & 11 & 4 \\
\hline
\end{tabular}

Tabla 6. Perímetro cefálico de escolares 6-10 años en relación a edad. Comuna de Colchane, 1982. 


\section{Comentario}

Los valores que entrega la variable perímetro cefálico siguen en general una tendencia ascendente con respecto a la edad. Esta característica se observa en ambos sexos. No se aprecian diferencias significativas al comparar ambas tablas.

\begin{tabular}{|l|c|c|c|c|c|c|}
\hline \multirow{2}{*}{ Edad (días) } & \multicolumn{2}{|c|}{ N } & \multicolumn{2}{c|}{ X (mm) } & \multicolumn{2}{c|}{ Ds } \\
\cline { 2 - 7 } & $\mathbf{M}$ & $\mathbf{F}$ & $\mathbf{M}$ & $\mathbf{F}$ & $\mathbf{M}$ & $\mathbf{F}$ \\
\hline $2190-2373$ & 4 & 6 & 241 & 236 & 21 & 21 \\
\hline $2374-2557$ & 9 & 7 & 236 & 232 & 11 & 16 \\
\hline $2558-2741$ & 6 & 13 & 254 & 237 & 11 & 27 \\
\hline $2742-2925$ & 11 & 7 & 260 & 256 & 18 & 8 \\
\hline $2926-3109$ & 2 & 5 & 259 & 255 & 2 & 6 \\
\hline $3110-3293$ & 10 & 8 & 263 & 251 & 7 & 34 \\
\hline $3294-3477$ & 5 & 5 & 262 & 253 & 10 & 10 \\
\hline $3478-3661$ & 10 & 7 & 273 & 273 & 10 & 10 \\
\hline $3662-3845$ & 7 & 5 & 274 & 294 & 20 & 31 \\
\hline $3846-4008$ & 8 & 2 & 285 & 295 & 20 & 4 \\
\hline
\end{tabular}

Tabla 7. Distancia biacromial en escolares 6-10 años en relación a edad. Comuna de Colchane, 1982.

\section{Comentario}

Se puede apreciar que los promedios para esta variable se presentan superiores en los individuos de sexo masculino en los primeros años escolares; a los 10 años esta característica se invierte, siendo superior a los promedios correspondientes a los individuos de sexo femenino.

\begin{tabular}{|l|c|c|c|c|c|c|}
\hline \multirow{2}{*}{ Edad (días) } & \multicolumn{2}{|c|}{ N } & \multicolumn{2}{c|}{ X (mm) } & \multicolumn{2}{c|}{ Ds } \\
\cline { 2 - 7 } & M & F & M & F & M & F \\
\hline $2190-2373$ & 4 & 6 & 183 & 192 & 7 & 14 \\
\hline $2374-2557$ & 9 & 7 & 187 & 188 & 11 & 17 \\
\hline $2558-2741$ & 6 & 13 & 194 & 188 & 5 & 12 \\
\hline $2742-2925$ & 11 & 7 & 199 & 197 & 12 & 12 \\
\hline $2926-3109$ & 2 & 5 & 187 & 184 & 5 & 9 \\
\hline $3110-3293$ & 10 & 8 & 198 & 188 & 10 & 12 \\
\hline $3294-3477$ & 5 & 7 & 197 & 192 & 11 & 21 \\
\hline $3478-3661$ & 10 & 5 & 208 & 209 & 11 & 10 \\
\hline $3662-3845$ & 7 & 2 & 202 & 221 & 12 & 17 \\
\hline $3846-4008$ & 8 & 2 & 224 & 220 & 24 & 5 \\
\hline
\end{tabular}

Tabla 8. Distancia bicrestal en escolares 6-10 años en relación a edad. Comuna de Colchane, 1982.

\section{Comentario}

En la Tabla 8 se observa un incremento paulatino de los valores de esta variable con respecto a la edad. Si se comparan los promedios por sexo se apreciará que éstos no presentan diferencias significativas. A los seis años la distancia bicrestal aparece superior para los individuos de sexo femenino.

\begin{tabular}{|l|c|c|c|c|c|c|}
\hline \multirow{2}{*}{ Edad (días) } & \multicolumn{2}{|c|}{ N } & \multicolumn{2}{c|}{ X (mm) } & \multicolumn{2}{c|}{ Ds } \\
\cline { 2 - 7 } & $\mathbf{M}$ & $\mathbf{F}$ & $\mathbf{M}$ & $\mathbf{F}$ & $\mathbf{M}$ & $\mathbf{F}$ \\
\hline $2190-2373$ & 4 & 6 & 190 & 175 & 5 & 8 \\
\hline $2374-2557$ & 9 & 7 & 191 & 185 & 11 & 10 \\
\hline $2558-2741$ & 6 & 13 & 188 & 182 & 10 & 6 \\
\hline $2742-2925$ & 11 & 7 & 194 & 189 & 7 & 7 \\
\hline $2926-3109$ & 2 & 5 & 195 & 190 & 6 & 4 \\
\hline $3110-3293$ & 10 & 8 & 203 & 196 & 7 & 12 \\
\hline $3294-3477$ & 5 & 5 & 201 & 188 & 11 & 2 \\
\hline $3478-3661$ & 10 & 7 & 200 & 192 & 8 & 9 \\
\hline $3662-3845$ & 7 & 5 & 201 & 191 & 11 & 9 \\
\hline $3846-4008$ & 8 & 2 & 204 & 199 & 12 & 1 \\
\hline
\end{tabular}

Tabla 9. Alto de cabeza en escolares 6-10 años en relación a edad. Comuna de Colchane, 1982.

\section{Comentario}

Los valores promedios para esta variable, en general siguen una tendencia ascendente con respecto a la edad en ambos sexos. Se observa que en esta población la variable altura de cabeza es superior en los individuos de sexo masculino.

\begin{tabular}{|l|c|c|c|c|c|c|}
\hline \multirow{2}{*}{ Edad (días) } & \multicolumn{2}{|c|}{ N } & \multicolumn{2}{c|}{ X (mm) } & \multicolumn{2}{c|}{ Ds } \\
\cline { 2 - 7 } & M & F & M & F & M & F \\
\hline $2190-2373$ & 4 & 6 & 602 & 606 & 17 & 14 \\
\hline $2374-2557$ & 9 & 7 & 607 & 600 & 15 & 25 \\
\hline $2558-2741$ & 6 & 13 & 609 & 607 & 16 & 24 \\
\hline $2742-2925$ & 11 & 7 & 637 & 636 & 17 & 25 \\
\hline $2926-3109$ & 2 & 5 & 617 & 620 & 16 & 8 \\
\hline $3110-3293$ & 10 & 8 & 652 & 659 & 28 & 17 \\
\hline $3294-3477$ & 5 & 5 & 671 & 656 & 41 & 36 \\
\hline $3478-3661$ & 10 & 7 & 668 & 655 & 17 & 25 \\
\hline $3662-3845$ & 7 & 5 & 674 & 661 & 29 & 37 \\
\hline $3846-4008$ & 8 & 2 & 689 & 704 & 29 & 16 \\
\hline
\end{tabular}

Tabla 10. Talla sentado en escolares 6-10 años en relación a edad. Comuna de Colchane, 1982. 


\section{Comentario}

Los valores promedios para esta variable siguen una tendencia ascendente en ambos sexos. Al comparar estos resultados se observa que a los seis y siete años los individuos masculinos presentan una medida promedio superior al sexo opuesto. A los ocho, nueve y 10 años esta peculiaridad aparece invertida.

\begin{tabular}{|l|c|c|c|c|c|}
\hline \multirow{2}{*}{ Edad (días) } & \multirow{2}{*}{$\mathbf{N}$} & \multicolumn{2}{|c|}{$\mathbf{X}(\mathbf{m m})$} & \multicolumn{2}{c|}{ Ds } \\
\cline { 3 - 6 } & & Der. & Izq. & Der. & Izq. \\
\hline $2190-2373$ & 4 & 457 & 465 & 51 & 27 \\
\hline $2374-2557$ & 9 & 480 & 462 & 24 & 24 \\
\hline $2558-2741$ & 6 & 501 & 480 & 11 & 25 \\
\hline $2742-2925$ & 11 & 505 & 504 & 36 & 26 \\
\hline $2926-3109$ & 2 & 492 & 504 & 8 & 22 \\
\hline $3110-3293$ & 10 & 541 & 523 & 29 & 20 \\
\hline $3294-3477$ & 5 & 543 & 520 & 18 & 29 \\
\hline $3478-3661$ & 10 & 550 & 549 & 26 & 30 \\
\hline $3662-3845$ & 7 & 570 & 561 & 38 & 32 \\
\hline $3846-4008$ & 8 & 569 & 594 & 39 & 4 \\
\hline
\end{tabular}

Tabla 11. Longitud total de miembro superior derecho-izquierdo en escolares sexo masculino en relación a edad. Comuna de Colchane, 1982.

\section{Comentario}

$\mathrm{Al}$ analizar los valores de esta tabla se pueden apreciar diferencias de longitud entre ellos: a los seis años el promedio del miembro superior derecho es $477.7 \mathrm{~mm}$; el promedio del miembro izquierdo es $466.6 \mathrm{~mm}$. A los siete años este valor es $503 \mathrm{~mm}$ para el derecho; $499 \mathrm{~mm}$ para el izquierdo. A los ocho años es $516.5 \mathrm{~mm}$ en el derecho, $513.5 \mathrm{en} \mathrm{el}$ izquierdo. A los nueve años el promedio es 546.5 $\mathrm{mm}$ en el derecho, 545.5 en el izquierdo. A los 10 años esta característica se invierte, siendo el miembro superior derecho de menor longitud $(569.5 \mathrm{~mm})$ que el miembro opuesto $(570.5 \mathrm{~mm})$.

\begin{tabular}{|l|c|c|c|c|c|}
\hline \multirow{2}{*}{ Edad (días) } & \multirow{2}{*}{$\mathbf{N}$} & \multicolumn{2}{|c|}{$\mathbf{X}(\mathbf{m m})$} & \multicolumn{2}{c|}{ Ds } \\
\cline { 3 - 6 } & & Der. & Izq. & Der. & Izq. \\
\hline $2190-2373$ & 6 & 463 & 465 & 25 & 27 \\
\hline $2374-2557$ & 7 & 467 & 462 & 23 & 24 \\
\hline $2558-2741$ & 13 & 486 & 480 & 26 & 25 \\
\hline $2742-2925$ & 7 & 504 & 504 & 36 & 26 \\
\hline $2926-3109$ & 5 & 495 & 504 & 13 & 22 \\
\hline $3110-3293$ & 8 & 526 & 523 & 19 & 20 \\
\hline $3294-3477$ & 5 & 535 & 520 & 25 & 29 \\
\hline $3478-3661$ & 7 & 539 & 549 & 22 & 30 \\
\hline $3662-3845$ & 5 & 566 & 561 & 31 & 32 \\
\hline $3846-4008$ & 2 & 600 & 594 & 10 & 4 \\
\hline
\end{tabular}

Tabla 12. Longitud total de miembro superior derecho-izquierdo en escolares sexo femenino en relación a edad. Comuna de Colchane, 1982.

\section{Comentario}

Las taxias de ambos segmentos ascienden a medida que se avanza en edad. A los seis años el miembro superior derecho tiene una medida promedio de $465 \mathrm{~mm}$, el izquierdo $463.5 \mathrm{~mm}$; a los siete años el miembro derecho mide $495 \mathrm{~mm}$, mientras que el izquierdo $492 \mathrm{~mm}$. A los ocho años el miembro superior derecho tiene un promedio de $510.5 \mathrm{~mm}$, el izquierdo $513.5 \mathrm{~mm}$. A los nueve años el miembro derecho mide $537 \mathrm{~mm}$ de promedio, el izquierdo $534.5 \mathrm{~mm}$. A la edad de 10 años esta medida es de $583 \mathrm{~mm}$ en el segmento derecho y $577.5 \mathrm{~mm}$ en el izquierdo.

\begin{tabular}{|l|c|c|c|c|c|}
\hline \multirow{2}{*}{ Edad (días) } & \multirow{2}{*}{$\mathbf{N}$} & \multicolumn{2}{|c|}{$\mathbf{X}(\mathbf{m m})$} & \multicolumn{2}{|c|}{ Ds } \\
\cline { 3 - 6 } & & Der. & Izq. & Der. & Izq. \\
\hline $2190-2373$ & 4 & 562 & 557 & 42 & 48 \\
\hline $2374-2557$ & 9 & 550 & 548 & 23 & 23 \\
\hline $2558-2741$ & 6 & 589 & 581 & 17 & 18 \\
\hline $2742-2925$ & 11 & 595 & 588 & 46 & 42 \\
\hline $2926-3109$ & 2 & 605 & 593 & 5 & 7 \\
\hline $3110-3293$ & 10 & 612 & 604 & 27 & 26 \\
\hline $3294-3477$ & 5 & 623 & 612 & 32 & 25 \\
\hline $3478-3661$ & 10 & 648 & 637 & 35 & 32 \\
\hline $3662-3845$ & 7 & 653 & 649 & 32 & 32 \\
\hline $3846-4008$ & 8 & 665 & 657 & 64 & 64 \\
\hline
\end{tabular}

Tabla 13. Longitud total de miembro inferior derecho-izquierdo en escolares masculino en relación a edad. Comuna de Colchane, 1982.

\section{Comentario}

Los miembros inferiores presentan valores promedios que aumentan en relación a la edad. En los varones se observa que el miembro inferior derecho presenta una medida promedio superior al segmento izquierdo: a los seis años el miembro derecho tiene un promedio de $556 \mathrm{~mm}$, el izquierdo $552 \mathrm{~mm}$; a los siete años el derecho mide $592 \mathrm{~mm}$ de promedio, mientras que el izquierdo $584.5 \mathrm{~mm}$; a los ocho años $608.5 \mathrm{~mm}$ el derecho y $598.5 \mathrm{~mm}$ el izquierdo; a los nueve años $635.5 \mathrm{~mm}$ el izquierdo, $624.5 \mathrm{~mm}$ el derecho; a los 10 años $659 \mathrm{~mm}$ el derecho, $653 \mathrm{~mm}$ el izquierdo. 


\begin{tabular}{|l|c|c|c|c|c|}
\hline \multirow{2}{*}{ Edad (días) } & \multirow{2}{*}{ N } & \multicolumn{2}{|c|}{ X (mm) } & \multicolumn{2}{c|}{ Ds } \\
\cline { 3 - 6 } & & Der. & Izq. & Der. & Izq. \\
\hline 2190 - 2373 & 6 & 557 & 552 & 38 & 28 \\
\hline $2374-2557$ & 7 & 545 & 536 & 32 & 32 \\
\hline $2558-2741$ & 13 & 561 & 556 & 34 & 32 \\
\hline $2742-2925$ & 7 & 577 & 578 & 37 & 30 \\
\hline $2926-3109$ & 5 & 579 & 577 & 10 & 15 \\
\hline $3110-3293$ & 8 & 618 & 610 & 22 & 26 \\
\hline $3294-3477$ & 5 & 632 & 629 & 45 & 44 \\
\hline $3478-3661$ & 7 & 650 & 636 & 29 & 29 \\
\hline $3662-3845$ & 5 & 678 & 671 & 50 & 46 \\
\hline $3846-4008$ & 2 & 727 & 717 & 7 & 7 \\
\hline
\end{tabular}

Tabla 14. Longitud total de miembro inferior derecho-izquierdo en escolares sexo femenino en relación a edad.

Comuna de Colchane, 1982.

\section{Comentario}

En general, en esta tabla se puede apreciar que el segmento inferior derecho presenta promedios mayores con respecto al izquierdo. A los seis años el miembro inferior derecho tiene un promedio de $551 \mathrm{~mm}$ contra 544.5 del izquierdo. A los siete años el segmento derecho mide $569 \mathrm{~mm}$ de promedio, el izquierdo $567 \mathrm{~mm}$. A los ocho años el derecho mide $598.5 \mathrm{~mm}$, el izquierdo 593.5. A los nueve años 641 $\mathrm{mm}$ el derecho, $632.5 \mathrm{~mm}$ el izquierdo. A los 10 años $702.5 \mathrm{~mm}$ contra $694 \mathrm{~mm}$ del izquierdo.

\section{Discusión}

El postulado general de esta investigación, en el plano metodológico, es el conocimiento antropomorfo del niño altiplánico. Este trabajo tiene un carácter preliminar. Es un estudio de casos de tipo descriptivo de validez local. Entrega datos antropométricos y antecedentes socioeconómicos, genéticos y de ingesta calórica. Estos antecedentes permiten homogeneizar la población en estudio, población que pertenece a un sector del Altiplano chileno.

Los resultados obtenidos, presentados en este informe, permitirán discernir el grado de variabilidad física de esta población escolar con respecto a otras poblaciones urbanas y/o rurales. Estas peculiaridades en el contexto de la morfología humana podrán ser analizadas en posteriores trabajos comparativos.
No se hace una discusión de la acción particular o conjunta de cada uno de los factores que inciden en la problemática planteada, porque la investigación del peso específico de estos factores en el estudio de la variabilidad humana requiere una implementación metodológica y práctica que escapa de los límites impuestos por los objetivos del trabajo, i.e., la descripción científica de algunos rasgos físicos de una población escolar altiplánica y algunas de sus concomitantes teóricas y metodológicas.

\section{Conclusiones}

1. Los niños escolares de la Comuna de Colchane habitan un territorio que se caracteriza por su altitud, en condiciones climáticas calificadas como rigurosas, donde se conjugan los elementos tradicionales que caracterizan la sociedad aymara con elementos culturales urbanos asimilados y derivados de la sociedad nacional.

2. El $95.6 \%$ de la población escolar de la Comuna tiene un aporte calórico promedio de $1601 \mathrm{kcal} /$ día.

3. El $98.5 \%$ de la población considerada tiene probabilidad de ser de ascendencia genética mixta. El $1.45 \%$ de ascendencia genética mongoloide. No se presentan individuos de ascendencia caucasoide.

4. El $100 \%$ de la población examinada tiene un nivel socioeconómico bajo, según la clasificación internacional de Graffar (1956).

5. Entre los seis y nueve años las taxias antropométricas de peso y talla tienden a ser mayores en los individuos de sexo masculino. Sin embargo, esta característica se invierte a la edad de 10 años.

6. En general, los valores de perímetro cafálico no presentan diferencias significativas al comparar los individuos por sexo.

7. La distancia biacromial es superior para los varones en los primeros años escolares. A los 10 años esta característica se invierte.

8. Los promedios de distancia bicrestal no presentan diferencias notables en ambos sexos. A los 6 años este valor es superior para los escolares de sexo femenino. 
9. En lo que respecta a la variable altura de cabeza, se observa que su valor promedio es superior para los individuos de sexo masculino.

10. La variable talla sentada en los individuos de sexo femenino presenta valores superiores a los ocho, nueve y 10 años de edad con respecto al sexo opuesto.

11. En los individuos masculinos la diferencia de longitud del miembro superior derecho, con respecto al izquierdo, se presenta con las siguientes características: a los seis años el miembro superior derecho es $12 \mathrm{~mm}$ más largo que el izquierdo; a los siete años $4 \mathrm{~mm}$; a los ocho años $3 \mathrm{~mm}$; a los nueve años $1 \mathrm{~mm}$ y a los 10 años $1 \mathrm{~mm}$.

12. En los individuos de sexo femenino la diferencia de longitud entre ambos segmentos superiores se presenta con las siguientes características: a los seis años el miembro superior derecho es $1.5 \mathrm{~mm}$ más largo que el izquierdo; a los siete años $3 \mathrm{~mm}$; a los 8 años $3.5 \mathrm{~mm}$; a los 9 años $2.5 \mathrm{~mm}$ y a los 10 años $5.5 \mathrm{~mm}$.

13. En los individuos de sexo masculino la diferencia de longitud del miembro inferior derecho con respecto al izquierdo se manifiesta de la siguiente manera: a los seis años el miembro inferior derecho es $4 \mathrm{~mm}$ más largo que el izquierdo; a los siete años $7.5 \mathrm{~mm}$; a los ocho años $10 \mathrm{~mm}$; a los nueve años $11 \mathrm{~mm}$ y a los 10 años $6 \mathrm{~mm}$.

14. En las mujeres la diferencia entre ambas extremidades inferiores se presenta con las características siguientes: a los seis años el miembro inferior derecho es $6.5 \mathrm{~mm}$ más largo que el izquierdo; a los siete años $2 \mathrm{~mm}$; a los ocho años $5 \mathrm{~mm}$; a los nueve años $8.5 \mathrm{~mm}$ y a los 10 años $8.5 \mathrm{~mm}$ más largo que el izquierdo.

\section{REFERENCIAS CITADAS}

GRAFFAR, M., 1956. Une méthode de classification sociale d'échantillons de population. Courrier VI.
PROVOSTE, P., 1977. Antecedentes socioeconómicos para el desarrollo del sector de Isluga. Centro Isluga de Investigaciones Andinas, Universidad del Norte, Iquique. 